\title{
Estimation of Unknown Functions of Iterative Difference Inequalities and Their Applications
}

\author{
Ricai Luo, ${ }^{1}$ Wu-Sheng Wang, ${ }^{2}$ and Honglei $\mathrm{Xu}^{3}$ \\ ${ }^{1}$ School of Computer and Information Engineering, Hechi University, Guangxi, Yizhou 546300, China \\ ${ }^{2}$ School of Mathematics and Statistics, Hechi University, Guangxi, Yizhou 546300, China \\ ${ }^{3}$ Department of Mathematics and Statistics, Curtin University, Perth, WA 6845, Australia
}

Correspondence should be addressed to Wu-Sheng Wang; wang4896@126.com and Honglei Xu; h.xu@curtin.edu.au

Received 28 March 2014; Accepted 10 July 2014; Published 22 July 2014

Academic Editor: Youjun Deng

Copyright (C) 2014 Ricai Luo et al. This is an open access article distributed under the Creative Commons Attribution License, which permits unrestricted use, distribution, and reproduction in any medium, provided the original work is properly cited.

We establish some new nonlinear retarded finite difference inequalities. The results that we propose here can be used as tools in the theory of certain new classes of finite difference equations in various difference situations. We also give applications of the inequalities to show the usefulness of our results.

\section{Introduction}

An integral inequality that provides an explicit bound to the unknown function furnishes a handy tool to investigate qualitative properties of solutions of differential and integral equations. One of the best known and widely used inequalities in the study of nonlinear differential equations is Gronwall-Bellman inequality $[1,2]$, which can be stated as follows. If $u$ and $f$ are nonnegative continuous functions on an interval $[a, b]$ satisfying

$$
u(t) \leq c+\int_{a}^{t} f(s) u(s) d s, \quad t \in[a, b],
$$

for some constant $c \geq 0$, then

$$
u(t) \leq c \exp \left(\int_{a}^{t} f(s) d s\right), \quad t \in[a, b] .
$$

Being an important tool in the study of qualitative properties of solutions of differential equations and integral equations, various generalizations of Gronwall inequalities $[1,2]$ and their applications have attracted great interests of many mathematicians [3-5]. Some recent works can be found in [612] and the references therein. Along with the development of the theory of integral inequalities and the theory of difference equations, more and more attentions are paid to discrete versions of Gronwall-type inequalities; see [13-36] and the references cited therein.

Sugiyama [13] established the most precise and complete discrete analogue of the Gronwall inequality [1] in the following form. Let $u(n)$ and $f(n)$ be nonnegative functions defined for $n \in \mathbf{N}$, and suppose that $f(n) \geq 0$ for every $n \in \mathbf{N}$. If

$$
u(n)<u_{0}+\sum_{s=n_{0}}^{n-1} f(s) u(s), \quad n \in \mathbf{N}
$$

where $\mathbf{N}$ is the set of points $n_{0}+k(k=0,1,2, \ldots), n_{0} \geq 0$ is a given integer, and $u_{0}$ is a nonnegative constant, then

$$
u(n)<u_{0} \prod_{s=n_{0}}^{n-1}[1+f(s)], \quad n \in \mathbf{N}
$$

Pachpatte [15] established some generalized discrete analogue of the Gronwall inequality in the following form. Let $m(s)$ be a positive and monotone nondecreasing function on $\mathbf{N}$, and let $a(s), b(s)$ be nonnegative functions on $\mathbf{N}$. If $u(n)$ satisfies

$$
u(n) \leq m(s)+\sum_{s=n_{0}}^{n-1} a(s)\left(u(s)+\sum_{\tau=n_{0}}^{s-1} b(\tau) u(\tau)\right), \quad \forall n \in \mathbf{N}
$$


then

$$
u(n) \leq P(n) m(s), \quad \forall n \in \mathbf{N},
$$

where

$$
P(n)=1+\sum_{s=n_{0}}^{n-1} a(s) \prod_{\tau=n_{0}}^{s-1}[1+a(\tau)+b(\tau)], \quad \forall n \in \mathbf{N}
$$

Lemma 1 (see [16]). Suppose that $u_{0}$ is a nonnegative constant and $u(n), a(n), b(n), c(n)$, and $d(n)$ are nonnegative functions defined on $\mathbf{N}, 1+a(n)-b(n) \geq 0$ for all $n \in \mathbf{N}$. If $u(n)$ satisfies the inequality

$$
\begin{aligned}
u(n) & \leq u_{0}+\sum_{s=n_{0}}^{n-1} a(s) u(s) \\
& +\sum_{s=n_{0}}^{n-1} b(s)\left(\sum_{\tau=n_{0}}^{s-1} c(\tau) u(\tau)\left(\sum_{t=n_{0}}^{\tau-1} d(t) u(t)\right)\right),
\end{aligned}
$$

$\forall n \in \mathbf{N}$,

then

$$
\begin{array}{r}
u(n) \leq u_{0} \prod_{s=n_{0}}^{n-1}[1+a(s)-b(s)] \\
+\sum_{s=n_{0}}^{n-1} b(s) R(s) \prod_{t=s+1}^{n-1}[1+a(t)-b(t)], \\
\forall n \in \mathbf{N},
\end{array}
$$

where

$$
\begin{aligned}
& R(n) \\
& \leq \frac{u_{0} \prod_{s=n_{0}}^{n-1}[1+a(s)+b(s)+c(s) Q(s)]}{1+u_{0} \sum_{s=n_{0}}^{n-1} c(s) \prod_{t=n_{0}}^{s}[1+a(t)+b(t)+c(t) Q(t)]}, \\
& \forall n \in \mathbf{N},
\end{aligned}
$$

in which

$Q(n)$

$$
\begin{array}{r}
\leq \frac{u_{0} \prod_{s=n_{0}}^{n-1}[1+a(s)+b(s)+d(s)]}{1-u_{0} \sum_{s=n_{0}}^{n-1} c(s) \prod_{t=n_{0}}^{s}[1+a(t)+b(t)+d(t)]}, \\
\forall n \in \mathbf{N},
\end{array}
$$

and $\sum_{s=n_{0}}^{n-1} c(s) \prod_{t=n_{0}}^{s}[1+a(t)+b(t)+d(t)]<u_{0}^{-1}$ for all $n \in \mathbf{N}$.

Lemma 2 (see $[14,18])$. Let $w(n, r)$ be a real-valued function defined for $n \in \mathbf{N}, 0 \leq r<\infty$ and monotone nondecreasing with respect to $r$ for any fixed $n \in \mathbf{N}$. Let $u(n)$ be a real-valued function defined for $n \in \mathbf{N}$ such that

$$
\Delta u(n) \leq w(n, u(n)), \quad \forall n \in \mathbf{N} .
$$

Let $r(n)$ be a solution of

$$
\Delta r(n)=w(n, r(n)), \quad r(0)=r_{0}, \quad \forall n \in \mathbf{N},
$$

such that $u(0) \leq r(0)$. Then

$$
u(n) \leq r(n), \quad \forall n \in \mathbf{N} .
$$

Pachpatte [18, 19] also established some difference inequalities of product form as follows. Let $u, a, b$ be nonnegative functions defined on $\mathbf{N}$ and let $c$ be a nonnegative constant. Let $w(n, r)$ be a nonnegative function defined for $n \in \mathbf{N}, 0 \leq r<\infty$ and monotone nondecreasing with respect to $r$ for any fixed $n \in \mathbf{N}$. If $u(n)$ satisfies

$$
\begin{array}{r}
u^{2}(n) \leq c^{2}+2 \sum_{s=n_{0}}^{n-1} u(s)\left[a(s)\left(u(s)+\sum_{t=n_{0}}^{s-1} b(t) u(t)\right)\right. \\
+w(s, u(s))], \quad \forall n \in \mathbf{N},
\end{array}
$$

then

$$
u(n) \leq P(n) r(n), \quad \forall n \in \mathbf{N},
$$

where $P(n)$ is defined by (7), and $r(n)$ is a solution of

$$
\Delta r(n)=w(n, P(n) r(n)), \quad r(0)=c, \quad \forall n \in \mathbf{N}
$$

Let $u, a, b$ be nonnegative functions defined for $n \in \mathbf{N}$ and let $c$ be a nonnegative constant. Let $w(n, r)$ be a nonnegative function defined for $n \in \mathbf{N}, 0 \leq r<\infty$ and monotone nondecreasing with respect to $r$ for any fixed $n \in \mathbf{N}$. If $u(n)$ satisfies

$$
\begin{aligned}
& u^{2}(n) \leq c^{2}+\sum_{s=n_{0}}^{n-1} a(s)(u(s+1)+u(s)) \\
& \times\left[\left(u(s)+\sum_{\tau=n_{0}}^{s-1} b(\tau) u(\tau)\right)\right. \\
& \\
& \\
&\quad+w(s, u(s))], \quad \forall n \in \mathbf{N},
\end{aligned}
$$

then

$$
u(n) \leq P(n) r(n), \quad \forall n \in \mathbf{N},
$$

where $P(n)$ is defined by (7), and $r(n)$ is a solution of the difference equation

$$
\Delta r(n)=a(n) w(n, P(n) r(n)), \quad r(0)=c, \quad \forall n \in \mathbf{N}
$$


Motivated by the results given in $[16,18,19]$, in this paper, we discuss new nonlinear finite difference inequalities:

$$
\begin{array}{r}
u^{2}(n) \leq c^{2}+\sum_{s=n_{0}}^{n-1} f(s)(u(s+1)+u(s)) \\
\times\left[\left(u(s)+\sum_{t=n_{0}}^{s-1} g(t) u(t)\right.\right. \\
\left.\quad \times \sum_{\tau=n_{0}}^{t-1} h(\tau) u(\tau)\right) \\
+w(s, u(s))], \quad \forall n \in \mathbf{N} .
\end{array}
$$

Our inequalities can be used as tools in the study of certain classes of finite difference equations. We also present some immediate applications to show the importance of our results to study the various problems in the theory of finite difference equations.

\section{Main Results}

Throughout this paper, let $\mathbf{R}=(-\infty,+\infty), \mathbf{R}_{+}=[0,+\infty)$. Let $\mathbf{N}:=\left\{n_{0}, n_{0}+1, n_{0}+2, \ldots\right\}$ and $\mathbf{N}_{T}:=\left\{n_{0}, n_{0}+1, n_{0}+\right.$ $2, \ldots, T\}, T \in \mathbf{N}$. For function $u(n), n \in \mathbf{N}$, we define the operator $\Delta$ by $\Delta u(n)=u(n+1)-u(n)$. Obviously, the linear difference equation $\Delta u(n)=f(n)$ with the initial condition $u\left(n_{0}\right)=0$ has the solution $u(n)=\sum_{s=n_{0}}^{n-1} f(s)$. For convenience, in the sequel we complementarily define that $\sum_{s=n_{0}}^{n_{0}-1} f(s)=0$ and $\prod_{s=n_{0}}^{n_{0}-1} f(s)=1$.

Theorem 3. Let $\beta>0$ be a constant, $x, y$ positive functions defined on $\mathbf{N}, x$ a monotone increasing function, and $y$ a monotone decreasing function. Let $\phi$ be a nonnegative function defined on $\mathbf{N}$ such that

$$
\begin{array}{r}
x(n+1) y(n+1)-x(n) y(n) \leq \phi(n)[x(n) y(n+1)]^{\beta}, \\
\forall n \in N .
\end{array}
$$

(i) Suppose $\beta>1$. If $1+(1-\beta)\left[x\left(n_{0}\right) y\left(n_{0}\right)\right]^{\beta-1} \sum_{s=n_{0}}^{n-1} \phi(s)$ $>0$, then

$$
x(n) \leq \frac{x\left(n_{0}\right) y\left(n_{0}\right) y(n)}{\left\{1+(1-\beta)\left[x\left(n_{0}\right) y\left(n_{0}\right)\right]^{\beta-1} \sum_{s=n_{0}}^{n-1} \phi(s)\right\}^{1 /(\beta-1)}},
$$

$\forall n \in N$

(ii) Suppose $0<\beta<1$. Then

$$
\begin{aligned}
x(n) \leq y^{-1}(n)\{[ & \left.x\left(n_{0}\right) y\left(n_{0}\right)\right]^{1-\beta} \\
& \left.+\sum_{s=n_{0}}^{n-1}(1-\beta) \phi(s)\right\}^{1 /(1-\beta)}, \quad \forall n \in N .
\end{aligned}
$$

Proof. (i) We apply mean value theorem for differentiation to the function

$$
F(z)=\frac{z^{1-\beta}}{(1-\beta)}, \quad z>0
$$

and then there exists $\xi$ between $x(n) y(n)$ and $x(n+1) y(n+1)$ such that

$$
\begin{aligned}
& {[x(n+1) y(n+1)]^{1-\beta}-[x(n) y(n)]^{1-\beta}} \\
& \quad=(1-\beta) \xi^{-\beta}[x(n+1) y(n+1)-x(n) y(n)] .
\end{aligned}
$$

Because $x(n)$ is monotone increasing and $y(n)$ is monotone decreasing and $-\beta<0$, we see that $[x(n) y(n+1)]^{-\beta} \geq[x(n+$ 1) $y(n+1)]^{-\beta}$ and $[x(n) y(n+1)]^{-\beta} \geq[x(n) y(n)]^{-\beta}$. So for all values of $\xi$ between $x(n) y(n)$ and $x(n+1) y(n+1)$ we have

$$
[x(n) y(n+1)]^{-\beta} \geq \xi^{-\beta} \text {. }
$$

From (22) and (27), we have

$$
\begin{aligned}
\xi^{-\beta} & {[x(n+1) y(n+1)-x(n) y(n)] } \\
\leq & {[x(n) y(n+1)]^{-\beta} \phi(n)[x(n) y(n+1)]^{\beta} } \\
& =\phi(n) .
\end{aligned}
$$

Since $1-\beta<0$, from (26) and (28) we have

$$
[x(n+1) y(n+1)]^{1-\beta}-[x(n) y(n)]^{1-\beta} \geq(1-\beta) \phi(n) .
$$

Taking $n=s$ in (29) and summing up over $s$ from $n_{0}$ to $n-1$, we obtain

$$
[x(n) y(n)]^{1-\beta} \geq\left[x\left(n_{0}\right) y\left(n_{0}\right)\right]^{1-\beta}+\sum_{s=n_{0}}^{n-1}(1-\beta) \phi(s) .
$$

From (30), we obtain our required estimation (23).

(ii) Now by following the same steps as in the proof of (i) before (29) we have

$$
[x(n+1) y(n+1)]^{1-\beta}-[x(n) y(n)]^{1-\beta} \leq(1-\beta) \phi(n),
$$

because $1-\beta>0$. Taking $n=s$ in (31) and summing up over $s$ from $n_{0}$ to $n-1$, we obtain

$$
[x(n) y(n)]^{1-\beta} \leq\left[x\left(n_{0}\right) y\left(n_{0}\right)\right]^{1-\beta}+\sum_{s=n_{0}}^{n-1}(1-\beta) \phi(s) .
$$

From (32), we obtain our required estimation (24). 
Theorem 4. Let $m(s)$ be a positive and monotone nondecreasing function defined on $\mathbf{N}$ and $f(s), g(s), h(s)$ nonnegative functions defined on $\mathbf{N}$. If $u(n)$ satisfies

$$
\begin{aligned}
& u(n) \leq m(n)+\sum_{s=n_{0}}^{n-1} f(s) u(s) \\
&+\sum_{s=n_{0}}^{n-1} f(s)\left(\sum_{t=n_{0}}^{s-1} g(t) u(t)\right. \\
&\left.\quad \times\left(\sum_{\tau=n_{0}}^{t-1} h(\tau) u(\tau)\right)\right),
\end{aligned}
$$$$
\forall n \in \mathbf{N} \text {, }
$$

then

$$
u(n) \leq m(n)+\sum_{s=n_{0}}^{n-1} f(s) U(s), \quad \forall n \in \mathbf{N}
$$

where

$$
U(n) \leq \frac{m(n) \prod_{s=n_{0}}^{n-1}[1+f(s)+g(s) V(s)]}{1+m(n) \sum_{s=n_{0}}^{n-1} g(s) \prod_{t=n_{0}}^{s}[1+f(t)+g(t) V(t)]},
$$

in which

$$
\begin{array}{r}
V(n)=\frac{m(n) \prod_{s=n_{0}}^{n-1}[1+f(s)+h(s)]}{1-m(n) \sum_{s=n_{0}}^{n-1} g(s) \prod_{t=n_{0}}^{s}[1+f(t)+h(t)]}, \\
\forall n \in \mathbf{N},
\end{array}
$$

and $\sum_{s=n_{0}}^{n-1} g(s) \prod_{t=n_{0}}^{s}[1+f(t)+h(t)]<m^{-1}(n)$ for all $n \in \mathbf{N}$.

Proof. Fix $T \in \mathbf{N}$, where $T$ is chosen arbitrarily, since $m(t)$ is a nonnegative and monotone nondecreasing function, from (33), we have

$$
\begin{aligned}
& u(n) \leq m(T)+\sum_{s=n_{0}}^{n-1} f(s) u(s) \\
&+\sum_{s=n_{0}}^{n-1} f(s)\left(\sum_{t=n_{0}}^{s-1} g(t) u(t)\right. \\
&\left.\times\left(\sum_{\tau=n_{0}}^{\mathrm{t}-1} h(\tau) u(\tau)\right)\right), \\
& \quad \forall n \in N_{T} .
\end{aligned}
$$

Define a function $z(n)$ by the right-hand side of (37). Then $z(n)$ is a positive and monotone nondecreasing function defined on $\mathbf{N}$. We have

$$
z\left(n_{0}\right)=m(T), \quad u(n) \leq z(n), \quad \forall n \in \mathbf{N} .
$$

Using the definitions of the operator $\Delta$ and $z$, we obtain

$$
\begin{aligned}
\Delta z(n) & =f(n)\left\{u(n)+\left[\sum_{t=n_{0}}^{n-1} g(t) u(t)\left(\sum_{\tau=n_{0}}^{t-1} h(\tau) u(\tau)\right)\right]\right\} \\
& \leq f(n)\left\{z(n)+\left[\sum_{t=n_{0}}^{n-1} g(t) z(t)\left(\sum_{\tau=n_{0}}^{t-1} h(\tau) z(\tau)\right)\right]\right\},
\end{aligned}
$$$$
\forall n \in N_{T}
$$

Let

$$
z_{1}(n)=z(n)+\left[\sum_{t=n_{0}}^{n-1} g(t) z(t)\left(\sum_{\tau=n_{0}}^{t-1} h(\tau) z(\tau)\right)\right]
$$

$$
\forall n \in N_{T}
$$

Then

$$
z_{1}\left(n_{0}\right)=z\left(n_{0}\right)=m(T), \quad z(n) \leq z_{1}(n), \quad \forall n \in N_{T} .
$$

It follows that

$$
\begin{aligned}
\Delta z_{1}(n) & =\Delta z(n)+g(n) z(n)\left(\sum_{\tau=n_{0}}^{n-1} h(\tau) z(\tau)\right) \\
& \leq f(n) z_{1}(n)+g(n) z_{1}(n)\left(\sum_{\tau=n_{0}}^{n-1} h(\tau) z_{1}(\tau)\right),
\end{aligned}
$$$$
\forall n \in N_{T} \text {. }
$$

Adding $g(n) z_{1}^{2}(n)$ to both sides of the above inequality we have

$$
\begin{array}{r}
\Delta z_{1}(n)+g(n) z_{1}^{2}(n) \leq f(n) z_{1}(n)+g(n) z_{1}(n) \\
\times\left[z_{1}(n)+\left(\sum_{\tau=n_{0}}^{n-1} h(\tau) z_{1}(\tau)\right)\right], \\
\forall n \in N_{T} .
\end{array}
$$

Put

$$
z_{2}(n)=z_{1}(n)+\sum_{\tau=n_{0}}^{n-1} h(\tau) z_{1}(\tau), \quad \forall n \in N_{T},
$$

and then $z_{1}(n) \leq z_{2}(n), z_{2}\left(n_{0}\right)=z_{1}\left(n_{0}\right)=m(T)$ and

$$
\begin{array}{r}
\Delta z_{2}(n)=\Delta z_{1}(n)+h(n) z_{1}(n) \\
\leq f(n) z_{2}(n)+g(n) z_{2}^{2}(n)+h(n) z_{2}(n), \\
\forall n \in N_{T} .
\end{array}
$$

We see that the inequality

$$
\begin{array}{r}
z_{2}(n+1)-(1+f(n)+h(n)) z_{2}(n) \leq g(n) z_{2}^{2}(n), \\
\forall n \in N_{T} .
\end{array}
$$


Define a function

$$
P_{1}(n)=\prod_{s=n_{0}}^{n-1}(1+f(s)+h(s))^{-1}, \quad \forall n \in N_{T} .
$$

Multiplying by $P_{1}(n+1)$ to both sides of (46) we obtain

$$
\begin{array}{r}
z_{2}(n+1) P_{1}(n+1)-z_{2}(n) P_{1}(n) \\
\leq P_{1}^{-1}(n+1) g(n)\left[z_{2}(n) P_{1}(n+1)\right]^{2}, \\
\forall n \in N_{T} .
\end{array}
$$

Let $x(n)=z_{2}(n), y(n)=P_{1}(n), \phi(n)=P_{1}^{-1}(n+1) g(n)$, and $\beta=$ 2. Because $z_{2}(n)$ is monotone increasing, $P_{1}(n)$ is monotone decreasing and $2>0$; applying Theorem 3 to (48) we obtain

$$
\begin{aligned}
& z_{2}(n) \leq \frac{z_{2}\left(n_{0}\right) P_{1}^{-1}(n)}{1-z_{2}\left(n_{0}\right) \sum_{s=n_{0}}^{n-1} g(s) P_{1}^{-1}(s+1)} \\
&=\frac{m(T) \prod_{s=n_{0}}^{n-1}[1+f(s)+h(s)]}{1-m(T) \sum_{s=n_{0}}^{n-1} g(s) \prod_{t=n_{0}}^{s}[1+f(t)+h(t)]}, \\
& \forall n \in N_{T},
\end{aligned}
$$

where $P_{1}\left(n_{0}\right)=1, z_{2}\left(n_{0}\right)=m(T)$ are used. Define a function $\widetilde{V}$ of the right-hand side of (49). Substituting (49) in (43) we obtain

$$
\begin{array}{r}
z_{1}(n+1)-(1+f(n)+g(n) \widetilde{V}(n)) z_{1}(n) \leq-g(n) z_{1}^{2}(n), \\
\forall n \in N_{T} .
\end{array}
$$

Performing the same derivation as in (46)-(49), we obtain from (50) that

$$
\begin{array}{r}
z_{1}(n) \leq \frac{m(T) \prod_{s=n_{0}}^{n-1}[1+f(s)+g(s) \widetilde{V}(s)]}{1+m(T) \sum_{s=n_{0}}^{n-1} g(s) \prod_{t=n_{0}}^{s}[1+f(t)+g(t) \widetilde{V}(t)]}, \\
\forall n \in N_{T} .
\end{array}
$$

Define a function $\widetilde{U}$ of the right-hand side of (51). Substituting (51) in (39) we obtain

$$
\Delta z(n)=f(n) \widetilde{U}(n), \quad \forall n \in N_{T} .
$$

Using (38), from (52) it follows that

$$
u(n) \leq m(T)+\sum_{s=n_{0}}^{n-1} f(s) \widetilde{U}(s), \quad \forall n \in N_{T} .
$$

Since $T \in \mathbf{N}$ is arbitrary, from (53), we get the required estimate (35).

Theorem 5. Let $u, f, g, h$ be nonnegative functions defined for $n \in \mathbf{N}$ and $c$ a nonnegative constant. Let $w(n, r)$ be a realvalued function defined for $n \in \mathbf{N}, 0 \leq r<\infty$, and monotone nondecreasing with respect to $r$ for any fixed $n \in \mathbf{N}$. If $u(n)$ satisfies (21), then

$$
u(n) \leq v(n)+\sum_{s=n_{0}}^{n-1} f(s) W_{1}(s), \quad \forall n \in \mathbf{N},
$$

where

$$
\begin{array}{r}
W_{1}(n) \\
\leq \frac{v(n) \prod_{s=n_{0}}^{n-1}\left[1+f(s)+g(s) W_{2}(s)\right]}{1+v(n) \sum_{s=n_{0}}^{n-1} g(s) \prod_{t=n_{0}}^{s}\left[1+f(t)+g(t) W_{2}(t)\right]}, \\
\forall n \in \mathbf{N},
\end{array}
$$

in which

$$
\begin{array}{r}
W_{2}(n)=\frac{v(n) \prod_{s=n_{0}}^{n-1}[1+f(s)+h(s)]}{1-v(n) \sum_{s=n_{0}}^{n-1} g(s) \prod_{t=n_{0}}^{s}[1+f(t)+h(t)]}, \\
\forall n \in \mathbf{N},
\end{array}
$$

and $v(n)$ is a solution of the difference equation

$$
\begin{gathered}
\Delta r(n)=f(n) w\left(n, r(n)+\sum_{s=n_{0}}^{n-1} f(s) W_{3}(s)\right), \\
r(0)=c, \quad \forall n \in \mathbf{N},
\end{gathered}
$$

where

$$
W_{3}(n) \leq \frac{r(n) \prod_{s=n_{0}}^{n-1}\left[1+f(s)+g(s) W_{4}(s)\right]}{1+r(n) \sum_{s=n_{0}}^{n-1} g(s) \prod_{t=n_{0}}^{s}\left[1+f(t)+g(t) W_{4}(t)\right]},
$$

in which

$$
W_{4}(n)=\frac{r(n) \prod_{s=n_{0}}^{n-1}[1+f(s)+h(s)]}{1-r(n) \sum_{s=n_{0}}^{n-1} g(s) \prod_{t=n_{0}}^{s}[1+f(t)+h(t)]},
$$

$\forall n \in \mathbf{N}$

and $\sum_{s=n_{0}}^{n-1} g(s) \prod_{t=n_{0}}^{s}[1+f(t)+h(t)]<v^{-1}(n)$ for all $n \in \mathbf{N}$.

Proof. We first assume that $c>0$ and define a function $z(n)$ by the right-hand side of (21). Then $z(n)$ is a positive and monotone nondecreasing function defined on $\mathbf{N}$. We have

$$
z(0)=c^{2}, \quad u(n) \leq \sqrt{z(n)}, \quad \forall n \in \mathbf{N} .
$$


Using the definitions of the operator $\Delta$ and $z$, we obtain

$$
\begin{aligned}
\Delta z(n)= & f(n)(u(n+1)+u(n)) \\
\times & {\left[\left(u(n)+\sum_{t=n_{0}}^{n-1} g(t) u(t) \sum_{\tau=n_{0}}^{t-1} h(\tau) u(\tau)\right)\right.} \\
& +w(n, u(n))] \\
\leq & f(n)(\sqrt{z(n+1)}+\sqrt{z(n)}) \\
\times & {\left[\left(\sqrt{z(n)}+\sum_{t=n_{0}}^{n-1} g(t) \sqrt{z(t)}\right.\right.} \\
& \left.\left.\times \sum_{\tau=n_{0}}^{t-1} h(\tau) \sqrt{z(\tau)}\right)+w(n, \sqrt{z(n)})\right],
\end{aligned}
$$

From (61) it follows that the inequality

$$
\begin{gathered}
\Delta(\sqrt{z(n)})=\frac{\Delta z(n)}{\sqrt{z(n+1)}+\sqrt{z(n)}} \\
\leq f(n)\left[\left(\sqrt{z(n)}+\sum_{t=n_{0}}^{n-1} g(t) \sqrt{z(t)}\right.\right. \\
\left.\quad \times \sum_{\tau=n_{0}}^{t-1} h(\tau) \sqrt{z(\tau)}\right) \\
+w(n, \sqrt{z(n)})]
\end{gathered}
$$

holds for all $n \in \mathbf{N}$. Setting $n=s$ in (62) and substituting $s=n_{0}, 1,2, \ldots, n-1$, successively, we get

$$
\begin{gathered}
\sqrt{z(n)} \leq c+\sum_{s=n_{0}}^{n-1} f(s) \\
\times\left[\left((\sqrt{z(s)})+\sum_{t=n_{0}}^{s-1} g(t) \sqrt{z(t)}\right.\right. \\
\left.\times \sum_{\tau=n_{0}}^{t-1} h(\tau) \sqrt{z(\tau)}\right) \\
+w(s, \sqrt{z(s)})]
\end{gathered}
$$

$\forall n \in \mathbf{N}$.

Define a function $z_{1}(n)$ by

$$
z_{1}(n)=c+\sum_{s=n_{0}}^{n-1} f(s) w(s, \sqrt{z(s)}), \quad \forall n \in \mathbf{N}
$$

Then $z_{1}(n)=c$ and

$$
\Delta z_{1}(n)=f(n) w(n, \sqrt{z(n)}), \quad \forall n \in \mathbf{N} .
$$

Using (64), the inequality (63) can be written as

$$
\begin{aligned}
& \sqrt{z(n)} \leq z_{1}(n) \\
&+\sum_{s=n_{0}}^{n-1} f(s)\left(\sqrt{z(s)}+\sum_{t=n_{0}}^{s-1} g(t) \sqrt{z(t)}\right. \\
&\left.\times \sum_{\tau=n_{0}}^{t-1} h(\tau) \sqrt{z(\tau)}\right), \\
& \forall n \in \mathbf{N} .
\end{aligned}
$$

Since $z_{1}(n)$ is positive and monotone nondecreasing for $n \in$ $\mathbf{N}, f(s), g(s), h(s)$ satisfy the conditions in Theorem 4 . Now an application of Theorem 4 to (66) yields

$$
\sqrt{z(n)} \leq z_{1}(n)+\sum_{s=n_{0}}^{n-1} f(s) \widetilde{W}_{1}(s), \quad \forall n \in \mathbf{N}
$$

where

$$
\begin{aligned}
& \widetilde{W}_{1}(n) \\
& \leq \frac{z_{1}(n) \prod_{s=n_{0}}^{n-1}\left[1+f(s)+g(s) \widetilde{W}_{2}(s)\right]}{1+z_{1}(n) \sum_{s=n_{0}}^{n-1} g(s) \prod_{t=n_{0}}^{s}\left[1+f(t)+g(t) \widetilde{W}_{2}(t)\right]}, \\
& \forall n \in \mathbf{N},
\end{aligned}
$$

in which

$$
\begin{array}{r}
\widetilde{W}_{2}(n)=\frac{z_{1}(n) \prod_{s=n_{0}}^{n-1}[1+f(s)+h(s)]}{1-z_{1}(n) \sum_{s=n_{0}}^{n-1} g(s) \prod_{t=n_{0}}^{s}[1+f(t)+h(t)]}, \\
\forall n \in \mathbf{N} .
\end{array}
$$

Since $w(n, r)$ is monotone nondecreasing with respect to $r$ for any fixed $n \in \mathbf{N}$, from (65) and (67), we have

$$
\Delta z_{1}(n) \leq f(n) w\left(n, z_{1}(n)+\sum_{s=n_{0}}^{n-1} f(s) \widetilde{W}_{1}(s)\right), \quad \forall n \in \mathbf{N}
$$

Now as a suitable application of Lemma 2, we obtain

$$
z_{1}(n) \leq v(n), \quad \forall n \in \mathbf{N}
$$

where $v(n)$ is a solution of (57). Using (60), (67), and (71), we obtain our required estimation (54).

If $c$ is nonnegative, we can carry out the above procedure with $c+\epsilon$ instead of $c$ where $\epsilon$ is an arbitrary small number. Letting $\epsilon \rightarrow 0$, we obtain (54). 


\section{Application to Finite Difference Equations}

In this section, we consider the following difference equation:

$$
\begin{aligned}
& \Delta x(n)= f(n) \\
& \times\left[F \left(n, x(n), \sum_{t=n_{0}}^{n-1} g(t) x(t)\right.\right. \\
&\left.\left.\times \sum_{\tau=n_{0}}^{t-1} H(t, \tau, x(\tau))\right)+K(n, x(n))\right], \\
& \forall n \in \mathbf{N}, \\
& x\left(n_{0}\right)=x_{0},
\end{aligned}
$$

where $F, H, K$ are real-valued functions defined, respectively, on $\mathbf{N} \times \mathbf{R}^{2}, \mathbf{N}^{2} \times \mathbf{R}, \mathbf{N} \times \mathbf{R}, f$ is as defined in Theorem 5 , and $x_{0}$ is a constant. We assume that

$$
\begin{gathered}
|K(n, x(n))| \leq w(n,|x(n)|), \\
|H(n, t, x(t))| \leq \sum_{t=n_{0}}^{n-1} h(t)|x(t)|, \\
|F(n, x(n), y(n))| \leq|x(n)|+|y(n)|,
\end{gathered}
$$

where $g, h, w$ are as defined in Theorem 5. Using the definitions of the operator $\Delta$, from (72), we see that the inequality

$$
\begin{aligned}
& x(n+1)-x(n) \\
&=f(n)\left[F \left(n, x(n), \sum_{t=n_{0}}^{n-1} g(t) x(t)\right.\right. \\
&\left.\left.\quad \times \sum_{\tau=n_{0}}^{t-1} H(t, \tau, x(\tau))\right)+K(n, x(n))\right]
\end{aligned}
$$

holds for all $n \in \mathbf{N}$. It follows that

$$
\begin{aligned}
& x^{2}(n+1)-x^{2}(n) \\
& =f(n)[x(n+1)-x(n)] \\
& \times\left[F\left(n, x(n), \sum_{t=n_{0}}^{n-1} g(t) x(t) \sum_{\tau=n_{0}}^{t-1} H(t, \tau, x(\tau))\right)\right. \\
& \quad+K(n, x(n))], \quad \forall n \in \mathbf{N} .
\end{aligned}
$$

From (76), we have

$$
\begin{aligned}
& x^{2}(n)=x^{2}\left(n_{0}\right)+\sum_{s=n_{0}}^{n-1} f(s)[x(s+1)-x(s)] \\
& \times\left[F \left(s, x(s), \sum_{t=n_{0}}^{s-1} g(t) x(t)\right.\right. \\
&\left.\quad \times \sum_{\tau=n_{0}}^{t-1} H(t, \tau, x(\tau))\right) \\
&+K(s, x(s))], \quad \forall n \in \mathbf{N} .
\end{aligned}
$$

Using the conditions (74), we obtain

$$
\begin{aligned}
|x(n)|^{2}=x^{2}\left(n_{0}\right)+\sum_{s=n_{0}}^{n-1} f(s)[|x(s+1)|-|x(s)|] & \\
\times & {\left[|x(s)|+\sum_{t=n_{0}}^{s-1} g(t)|x(t)|\right.} \\
& \times \sum_{\tau=n_{0}}^{t-1} h(\tau)|x(\tau)| \\
& +w(s,|x(s)|)], \quad \forall n \in \mathbf{N} .
\end{aligned}
$$

Now an application of Theorem 5 to (78) yields the estimation of the difference equation (72), that is,

$$
|x(n)| \leq v(n)+\sum_{s=n_{0}}^{n-1} f(s) W_{5}(s), \quad \forall n \in \mathbf{N},
$$

where

$W_{5}(n) \leq \frac{v(n) \prod_{s=n_{0}}^{n-1}\left[1+f(s)+g(s) W_{6}(s)\right]}{1+v(n) \sum_{s=n_{0}}^{n-1} g(s) \prod_{t=n_{0}}^{s}\left[1+f(t)+g(t) W_{6}(t)\right]}$, $\forall n \in \mathbf{N}$

in which

$$
\begin{array}{r}
W_{6}(n)=\frac{v(n) \prod_{s=n_{0}}^{n-1}[1+f(s)+h(s)]}{1-v(n) \sum_{s=n_{0}}^{n-1} g(s) \prod_{t=n_{0}}^{s}[1+f(t)+h(t)]}, \\
\forall n \in \mathbf{N},
\end{array}
$$

and $v(n)$ is a solution of the difference equation

$$
\begin{gathered}
\Delta r(n)=f(n) w\left(n, r(n)+\sum_{s=n_{0}}^{n-1} f(s) W_{7}(s)\right), \\
r(0)=\left|x_{0}\right|, \quad \forall n \in \mathbf{N},
\end{gathered}
$$


where

$$
\begin{array}{r}
W_{7}(n) \leq \frac{r(n) \prod_{s=n_{0}}^{n-1}\left[1+f(s)+g(s) W_{8}(s)\right]}{1+r(n) \sum_{s=n_{0}}^{n-1} g(s) \prod_{t=n_{0}}^{s}\left[1+f(t)+g(t) W_{8}(t)\right]}, \\
\forall n \in \mathbf{N},
\end{array}
$$

in which

$$
\begin{array}{r}
W_{8}(n)=\frac{r(n) \prod_{s=n_{0}}^{n-1}[1+f(s)+h(s)]}{1-r(n) \sum_{s=n_{0}}^{n-1} g(s) \prod_{t=n_{0}}^{s}[1+f(t)+h(t)]}, \\
\forall n \in \mathbf{N},
\end{array}
$$

and $\sum_{s=n_{0}}^{n-1} g(s) \prod_{t=n_{0}}^{s}[1+f(t)+h(t)]<v^{-1}(n)$ for all $n \in \mathbf{N}$.

\section{Conflict of Interests}

The authors declare that there is no conflict of interests regarding the publication of this paper.

\section{Acknowledgments}

This research was supported by National Natural Science Foundation of China (Project no. 11161018,11171079), the NSF of Guangxi Zhuang Autonomous Region (no. 2012GXNSFAA053009), and the SRF of the Education Department of Guangxi Zhuang Autonomous Region (nos. 201106LX599 and 201106LX591).

\section{References}

[1] T. H. Gronwall, "Note on the derivatives with respect to a parameter of the solutions of a system of differential equations," Annals of Mathematics: Second Series, vol. 20, no. 4, pp. 292-296, 1919.

[2] R. Bellman, "The stability of solutions of linear differential equations," Duke Mathematical Journal, vol. 10, pp. 643-647, 1943.

[3] D. S. Mitrinović, J. E. Pečarić, and A. M. Fink, Inequalities Involving Functions and Their Integrals and Derivatives, vol. 53 of Mathematics and Its Applications, Kluwer Academic Publishers, Dordrecht, The Netherlands, 1991.

[4] D. Bainov and P. Simeonov, Integral Inequalities and Applications, vol. 57 of Mathematics and its Applications (East European Series), Kluwer Academic, Dordrecht, The Netherlands, 1992.

[5] B. G. Pachpatte, Inequalities for Differential and Integral Equations, vol. 197, Academic Press, New York, NY, USA, 1998.

[6] R. P. Agarwal, S. Deng, and W. Zhang, "Generalization of a retarded Gronwall-like inequality and its applications," Applied Mathematics and Computation, vol. 165, no. 3, pp. 599-612, 2005.

[7] O. Lipovan, "Integral inequalities for retarded Volterra equations," Journal of Mathematical Analysis and Applications, vol. 322, no. 1, pp. 349-358, 2006.

[8] W. Cheung, "Some new nonlinear inequalities and applications to boundary value problems," Nonlinear Analysis. Theory, Methods \& Applications, vol. 64, no. 9, pp. 2112-2128, 2006.
[9] R. P. Agarwal, C. S. Ryoo, and Y. Kim, "New integral inequalities for iterated integrals with applications," Journal of Inequalities and Applications, vol. 2007, Article ID 24385, 18 pages, 2007.

[10] W. Wang, "A generalized retarded Gronwall-like inequality in two variables and applications to BVP," Applied Mathematics and Computation, vol. 191, no. 1, pp. 144-154, 2007.

[11] A. Abdeldaim and M. Yakout, "On some new integral inequalities of Gronwall-Bellman-Pachpatte type," Applied Mathematics and Computation, vol. 217, no. 20, pp. 7887-7899, 2011.

[12] W. S. Wang, D. Huang, and X. Li, "Generalized retarded nonlinear integral inequalities involving iterated integrals and an application," Journal of Inequalities and Applications, vol. 2013, article 376, 2013.

[13] S. Sugiyama, "On the stability problems of difference equations," Bulletin of the Science and Engineering Research Laboratory, Waseda University, vol. 45, pp. 140-144, 1969.

[14] B. G. Pachpatte, "Finite-difference inequalities and an extension of Lyapunov's method," The Michigan Mathematical Journal, vol. 18, pp. 385-391, 1971.

[15] B. G. Pachpatte, "On some new discrete inequalities and their applications," Proceedings of the National Academy of Sciences, India, vol. 46, no. 4, pp. 255-262, 1976.

[16] B. G. Pachpatte, "On discrete inequalities related to Gronwall's inequality," Proceedings of the Indian Academy of Science A, vol. 85, no. 1, pp. 26-40, 1977.

[17] B. G. Pachpatte, "Finite difference inequalities and discrete time control systems," Indian Journal of Pure and Applied Mathematics, vol. 9, no. 12, pp. 1282-1290, 1978.

[18] B. G. Pachpatte, "Comparison theorems related to a certain inequality used in the theory of differential equations," Soochow Journal of Mathematics, vol. 22, no. 3, pp. 383-394, 1996.

[19] B. G. Pachpatte, "Inequalities applicable in the theory of finite difference equations," Journal of Mathematical Analysis and Applications, vol. 222, no. 2, pp. 438-459, 1998.

[20] E. Yang, "A new nonlinear discrete inequality and its application," Annals of Differential Equations: Weifen Fangcheng Niankan, vol. 17, no. 3, pp. 261-267, 2001.

[21] B. G. Pachpatte, "On some fundamental integral inequalities and their discrete analogues," Journal of Inequalities in Pure and Applied Mathematics, vol. 2, no. 2, article 15, 2001.

[22] F. W. Meng and W. N. Li, "On some new nonlinear discrete inequalities and their applications," Journal of Computational and Applied Mathematics, vol. 158, no. 2, pp. 407-417, 2003.

[23] W.-S. Cheung and J. Ren, "Discrete non-linear inequalities and applications to boundary value problems," Journal of Mathematical Analysis and Applications, vol. 319, no. 2, pp. 708-724, 2006.

[24] B. G. Pachpatte, Integral and Finite Difference Inequalities and Applications, vol. 205 of North-Holland Mathematics Studies, Elsevier Science, Amsterdam, The Netherlands, 2006.

[25] W. Sheng and W. N. Li, "Bounds on certain nonlinear discrete inequalities," Journal of Mathematical Inequalities, vol. 2, no. 2, pp. 279-286, 2008.

[26] Q.-H. Ma and W.-S. Cheung, "Some new nonlinear difference inequalities and their applications," Journal of Computational and Applied Mathematics, vol. 202, no. 2, pp. 339-351, 2007.

[27] Y. J. Cho, S. S. Dragomir, and Y. Kim, "On some integral inequalities with iterated integrals," Journal of the Korean Mathematical Society, vol. 43, no. 3, pp. 563-578, 2006.

[28] W. Wang, "A generalized sum-difference inequality and applications to partial difference equations," Advances in Difference Equations, vol. 2008, article 12, Article ID 695495, 2008. 
[29] W.-S. Wang, "Estimation on certain nonlinear discrete inequality and applications to boundary value problem," Advances in Difference Equations, vol. 2009, Article ID 708587, 8 pages, 2009.

[30] K.-L. Zheng, S.-M. Zhong, and M. Ye, "Discrete nonlinear inequalities in time control systems," in Proceedings of the International Conference on Apperceiving Computing and Intelligence Analysis (ICACIA '09), pp. 403-406, Chengdu, China, October 2009.

[31] W. S. Wang, Z. Li, and W. S. Cheung, "Some new nonlinear retarded sum-difference inequalities with applications," Advances in Difference Equations, vol. 2011, no. 1, article 41, 2011.

[32] H. Zhou, D. Huang, W.-S. Wang, and J.-X. Xu, "Some new difference inequalities and an application to discrete-time control systems," Journal of Applied Mathematics, vol. 2012, Article ID 214609, 14 pages, 2012.

[33] Q. He, T. Sun, and H. Xi, "Dynamics of a family of nonlinear delay difference equations," Abstract and Applied Analysis, vol. 2013, Article ID 456530, 4 pages, 2013.

[34] Z.-X. Chen and K. H. Shon, "Fixed points of meromorphic solutions for some difference equations," Abstract and Applied Analysis, vol. 2013, Article ID 496096, 7 pages, 2013.

[35] W. S. Wang and S. Wu, "Some difference inequalities for iterated sums with applications," Abstract and Applied Analysis, vol. 2013, Article ID 804152, 10 pages, 2013.

[36] Y. Qin and W. Wang, "A generalized nonlinear sum-difference inequality of product form," Journal of Applied Mathematics, vol. 2013, Article ID 247585, 7 pages, 2013. 


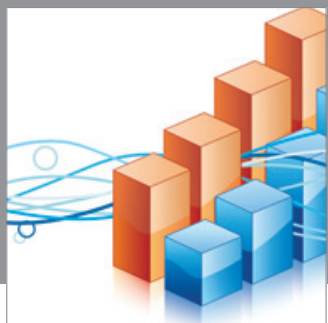

Advances in

Operations Research

mansans

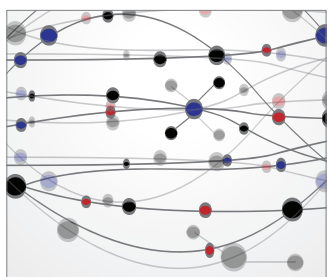

The Scientific World Journal
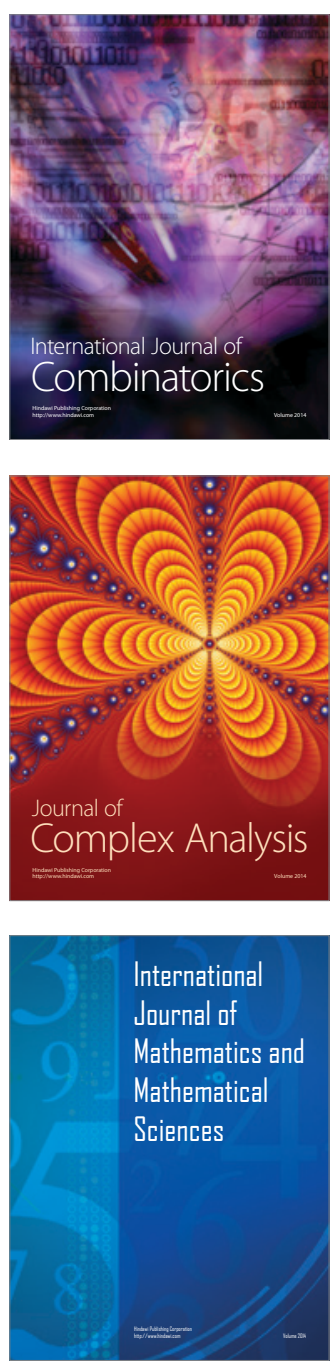
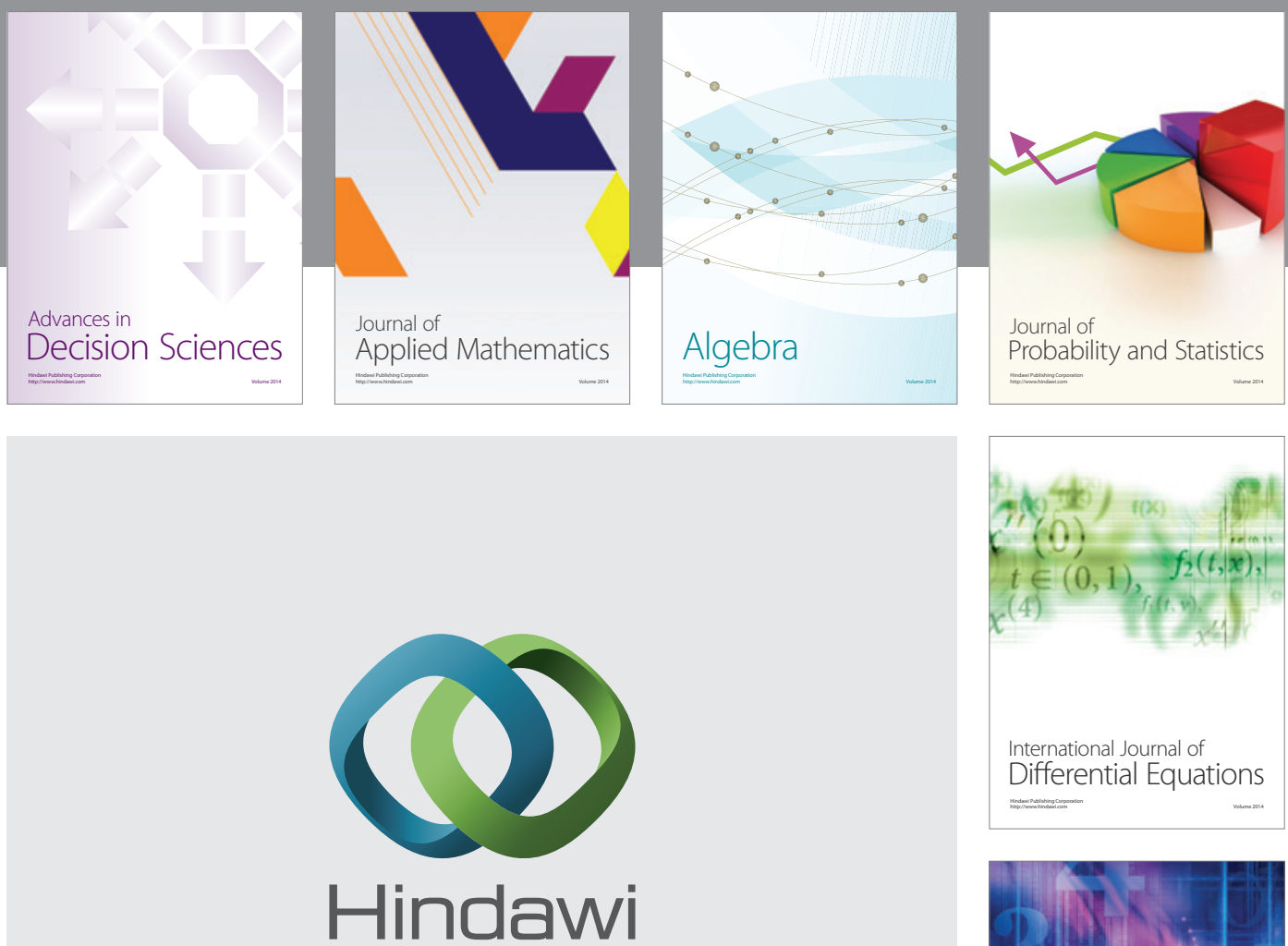

Submit your manuscripts at http://www.hindawi.com
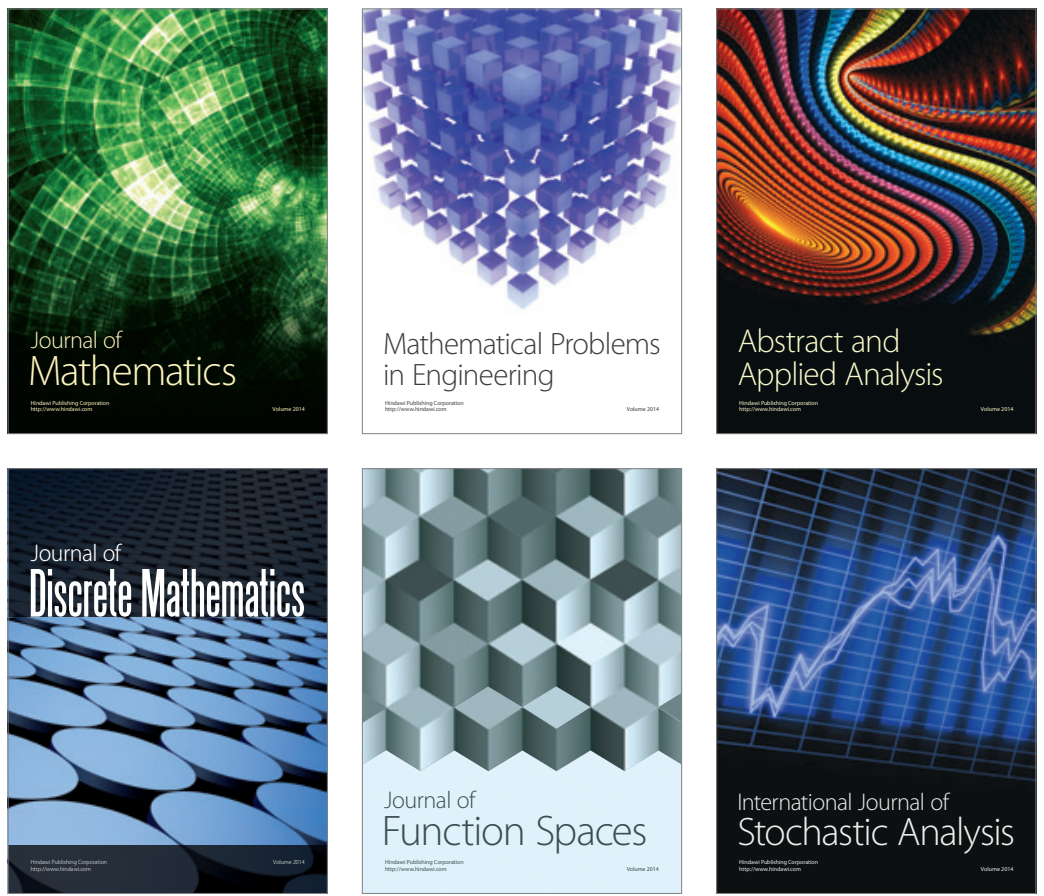

Journal of

Function Spaces

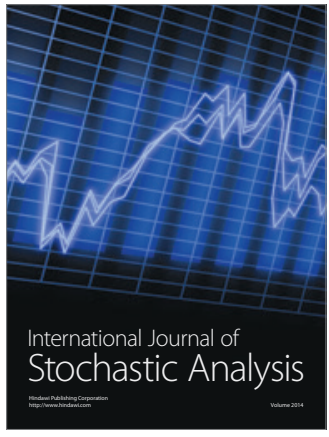

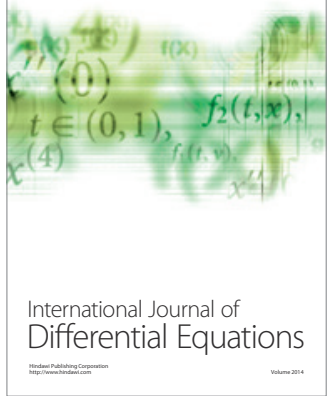
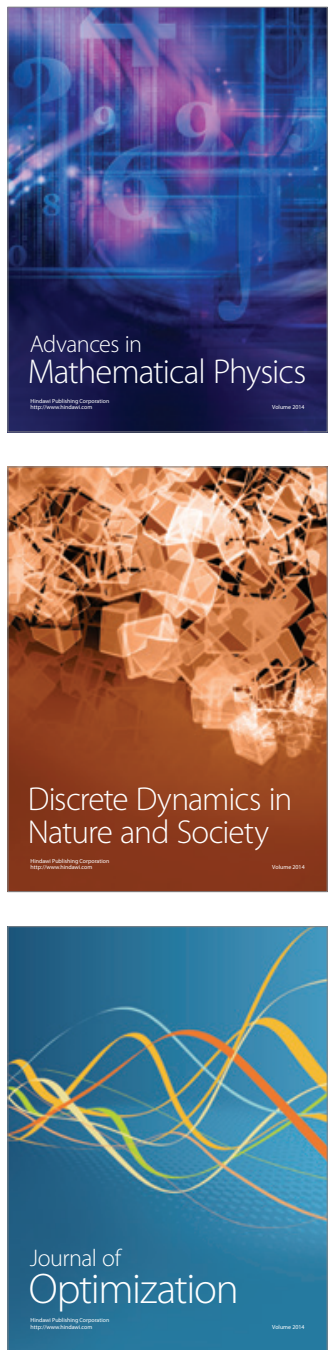